\title{
CORRECTION
}

Check for updates

Cite this: Mater. Chem. Front., 2018, 2, 2340

DOI: $10.1039 / c 8 q m 90048 e$

rsc.li/frontiers-materials

\section{Correction: Reticular control of interpenetration in a complex metal-organic framework}

\author{
Trang T. M. Nguyen, ${ }^{a}$ Hung M. Le, ${ }^{a}$ Yoshiyuki Kawazoe ${ }^{\mathrm{b}}$ and Ha L. Nguyen*ac \\ Correction for 'Reticular control of interpenetration in a complex metal-organic framework' by \\ Trang T. M. Nguyen et al., Mater. Chem. Front., 2018, DOI: 10.1039/c8qm00368h.
}

The authors regret that the journal name in reference 19 was not correct. The correct citation, now also updated with a DOI, is as follows: H. L. Nguyen, T. T. Vu, D.-K. Nguyen, C. A. Trickett, T. L. H. Doan, C. S. Diercks, V. Q. Nguyen and K. E. Cordova, Commun. Chem., 2018, DOI: 10.1038/s42004-018-0071-6.

The Royal Society of Chemistry apologises for these errors and any consequent inconvenience to authors and readers.

\footnotetext{
${ }^{a}$ Center for Innovative Materials and Architectures (INOMAR), Vietnam National University (VNUHCM), Ho Chi Minh City, 721337, Vietnam. E-mail: nlha@inomar.edu.vn ${ }^{b}$ New Industry Creation Hatchery Center, Tohoku University, Sendai, 980-8579, Japan

${ }^{c}$ Center for Research Excellence in Nanotechnology (CENT), King Fahd University of Petroleum and Minerals, Dhahran 34464, Saudi Arabia
} 